\title{
Hydrogen Cryomagnetics for Decentralised Energy Management and Superconductivity
}

\author{
B. A. Glowacki • W. J. Nuttall • E. Hanley • \\ L. Kennedy • D. O'Flynn
}

Received: 19 June 2014 / Accepted: 30 July 2014 / Published online: 30 August 2014

(C) The Author(s) 2014. This article is published with open access at Springerlink.com

\begin{abstract}
As we enter the second century of superconductivity, helium still prevails as the cryogenic coolant of choice. What does the future of helium hold? What can be done to avoid the squandering of this precious resource? In our presentation, we will discuss the use of cryogenic hydrogen originated from renewable and low- $\mathrm{CO}_{2}$ emission sources. We suggest that $20 \mathrm{~K}$ of liquid hydrogen can ultimately displace helium as an indirect coolant in a range of superconducting electromagnetic devices. As is already well documented, superconductors have much potential underpinning the future developments in transportation, energy supply/storage and also in medical applications. Although superconductors that can operate at liquid hydrogen temperatures, such as $\mathrm{MgB}_{2}$ and $\mathrm{YBa}_{2} \mathrm{Cu}_{3} \mathrm{O}_{7}$, are not yet truly commercially available, research indicates that these will be feasible in the near future.
\end{abstract}

Keywords Superconductivity · Hydrogen cryomagnetics . Decentralised hydrogen production

\footnotetext{
B. A. Glowacki $(\bowtie)$

Department of Materials Science and Metallurgy,

University of Cambridge, Room 1-027, 27 Charles

Babbage Road, Cambridge CB3 0FS, UK

e-mail: bag10@cam.ac.uk
}

B. A. Glowacki · E. Hanley · L. Kennedy · D. O’Flynn Department of Physics and Energy, University of Limerick, Castletroy, Limerick, Ireland

B. A. Glowacki

Institute of Power Engineering, Mory 8, 01-330 Warsaw, Poland

W. J. Nuttall

Department of Engineering and Innovation, The Open University, Milton Keynes, Buckinghamshire, MK7 6AA, UK

\section{Introduction to the Hydrogen Market}

In this article, we will try to explain that superconductivity interlocked with the hydrogen economy can be the solution to our decentralised energy problems.

The world is encountering a continuous depletion of fossil fuel resources and an acceleration of climate change, widely believed to be caused by the emission of greenhouse gases. Energy demand is also expected to increase by $56 \%$ between 2010 and 2040 [1], increasing by a factor of 5 by 2100 due to human population growth and accelerated global industrialisation. Considering the projected increased energy demand in transportation and electrical supply, there will be an increased pressure on energy generation, storage and use and changes will have to be made. The energy supply is currently in transition partly due to the need for a lower carbon future. Due to the expected increase in the penetration of intermittent renewable energy [2] to the electrical grid in the future, there is a need for reliable energy storage systems. The research and development of these systems focuses on reducing the effects of the world's emerging energy problems. The integration of energy storage systems with renewable energy, such as wind, would allow otherwise wasted energy to be stored. Capital costs of storage systems are a barrier to their widespread installation. However, with energy management, profits can be enhanced by supplying stored energy at times when demand and prices are high. Increased storage capacity is necessary as the use of renewable energy increases. Pumped hydroelectric storage (PHES) currently represents around $99 \%$ of the global energy storage capacity with an installed capacity of $127,000 \mathrm{MW}$. The remaining $1 \%$ of storage capacity is divided among other storage technologies shown in Fig.1a [3]. What can be the most surprising conclusion drawn from Fig. 1a is that hydrogen storage 
Fig. 1 a) Non-PHES installed storage capacity accounting for $1 \%$ of worldwide storage capacity [3](PHES is constrained as it is site specific so there is a need for renewable energy storage methods that are modular and can be installed at most sites); b) Global hydrogen use [4]

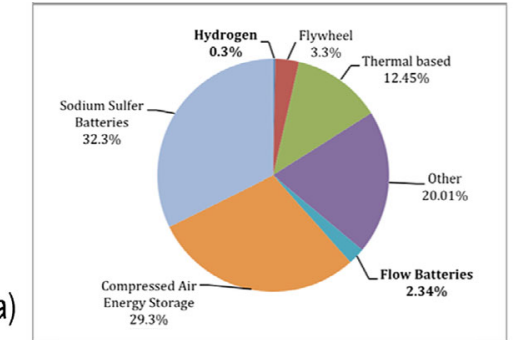

b)

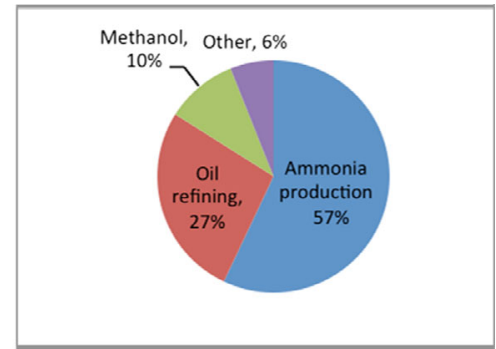

constitute for $0.3 \%$ of the remaining storage capacity whereas production of hydrogen is growing strongly (see Fig. 2).

Global hydrogen production was estimated to be 53 million tonnes in 2010, and this demand is expected to grow by an average of $5.6 \%$ in the coming years [4]. Hydrogen applications include a major role as a chemical feedstock, as shown in Fig. 1b. It is anticipated that the drivers for increased hydrogen production will continue to be regulations requiring lower sulphur levels in petroleum products, the need to process crude oils of decreasing quality and the search for cleaner fuel options. Merchant hydrogen production is a small segment but it will be the fastest growing

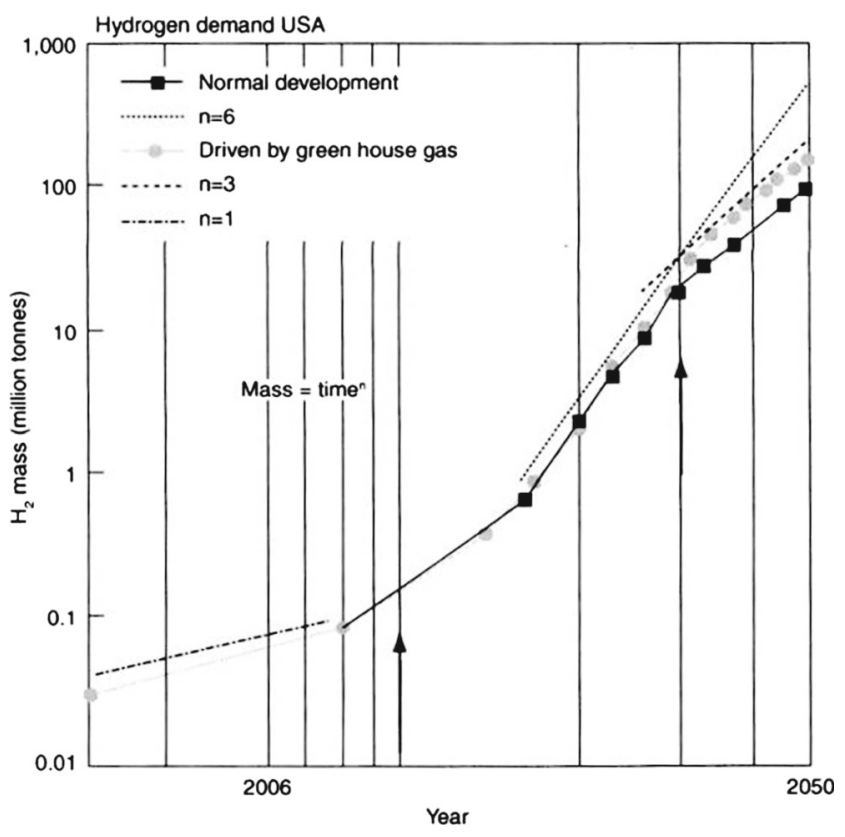

Fig. 2 Predicted mass production demanded by the USA economy versus time. It is evident that the differences for two scenarios, normal development and demand driven by greenhouse gas, are almost identical until 2050. The $n$ value of the calculated mass $=$ time $^{n}$ may increase from 2 to 6 over the next 10 years segment in the future. At present it accounts for only 12 $\%$ of total on-purpose hydrogen generation but this share is estimated to increase up to $16 \%$ by 2016 [5].

\section{Hydrogen Storage}

Hydrogen is an energy carrier with a low volumetric energy density at standard atmospheric pressure and temperature. For this reason, it must be stored as compressed hydrogen usually at 35 or $70 \mathrm{MPa}$ or as liquid hydrogen in cryogenic containers. The hydrogen storage system requires the use of another feedstock to produce hydrogen as the storage medium. The most common electrolysers are alkaline electrolysers, and efficiencies range between 52 and $83 \%$ [6]. Polymer electrolyte membrane electrolysers [7] are being developed and are expected to have increased operational efficiencies and could potentially lower the overall costs of the electrolyser. Different efficiencies were stated for the compression of hydrogen ranging from low efficiencies of $52 \%$ [8] to high efficiencies of $85 \%$ [9].

There are different types of fuel cells available. The proton exchange membrane fuel cell (PEMFC) is the most advanced for transport and stationery applications and has an efficiency of $53.5 \%$ [8]. Another fuel cell that is in the research phase is the solid oxide fuel cell [10] (SOFC) which is estimated to have higher efficiencies due to high operating temperatures in which some of the heat can be used to supply heat for domestic purposes. They can be used for the co-production of renewable heat and electricity. Compressed hydrogen storage is suitable for wind energy storage due to its high energy density [11] of $1,246 \mathrm{kWh} / \mathrm{m}^{3}$. This makes it suitable for bulk energy storage.

Drawing upon much experience in superconductivity, we suggest that hydrogen can also be stored as liquid at temperatures ranging from 14 to $33 \mathrm{~K}$. An important application of liquid hydrogen is as a cryogenic coolant for almost all technical superconductors. The cost of liquefaction of hydrogen depends on the quantity of hydrogen being 
produced. As the quantity of hydrogen is increased, less energy is wasted and the more efficient and cost-effective the process. Large quantities of energy must be available for the liquefaction process. The liquefaction process can occur by the Joule-Thomson expansion cycle [12]. The hydrogen is compressed at ambient pressure and passed through a heat exchanger in which the temperature is reduced. As a result of hydrogen cooling on expansion, the temperature should be below the inversion temperature $T_{\mathrm{inv}}=$ $200 \mathrm{~K}$. A nitrogen precooling step is introduced before the hydrogen is passed to the expansion valve. The energy required for the compressor and expansion valves reduces the overall efficiency of the process. As liquid hydrogen is a cryogenic liquid with a low boiling temperature of $T_{\text {boil }}=20 \mathrm{~K}$ (under normal pressure), it must be stored in insulated cryogenic containers which are designed with double walls and an insulating space between the two walls to reduce heat transfer to the liquid. Heat transfer causes the liquid to evaporate and form gas a process called boil off. Heat also arises from the ortho-para conversion of hydrogen. Hydrogen can exist as ortho- or para-hydrogen depending on electron configuration but the ortho-para conversion causes the release of heat resulting in boil off. At room temperature, hydrogen consists of $25 \%$ para- and $75 \%$ ortho-hydrogen, whereas at $20 \mathrm{~K}$, approximately $99 \%$ is para-hydrogen. To minimise boil off of the hydrogen for longer storage, an ortho-para conversion must be completed before liquefaction. The use of catalysts facilitates for the ortho-para conversion of hydrogen [12].

Another type of energy storage that utilises hydrogen and is proposed to be a flexible storage option for backup electricity [13] is the superconducting magnetic energy storage (SMES) system which has the potential to benefit greatly from hydrogen cryomagnetic technology. The proposed electricity to be used is intermittent energy from renewable generation. Liquid hydrogen is crucial for the system as it is used in the fuel cell to provide electricity and also to cool the superconducting coil. An oxygen tank is required for the system. It is suggested that the fuel cell is used to supply constant electricity and the SMES is used to respond to fluctuations at peak times or power cuts. With the increasing penetration of renewable technologies into the grid, there is a need for large-scale storage of electricity. The proposed LIQHYSMES system is a hybrid system using liquid hydrogen and a superconducting coil; the coil used is a magnesium boride coil. This particular model has the capacity of $12 \mathrm{GWh}$ of energy in the liquid hydrogen and 48 GJ stored in the SMES [14].

It is widely anticipated that hydrogen will be used to complement electricity in the future energy scenario of the world and act as a storage and cryogenic coolant.

\section{Synergy Between Superconductivity and Liquid Hydrogen}

The predicted and well-documented incoming shortage of helium for superconducting applications has been presented already in open literature $[15,16]$. On the other hand, use of hydrogen as a cryogenic coolant has been envisaged as a viable and more economically justified cooling option for superconducting devices [17]. Novel engineering designs made possible using medium-temperature $\mathrm{MgB}_{2}$ superconducting wires, as developed originally in Cambridge [18], include the following: a self-contained fully electric superconducting ship, DC fault current limiters, high DC current homopolar motors [19], cheaper superconducting $\mathrm{MgB}_{2}$ magnets for fusion [20], SMES [21] and magnetic resonance imaging (MRI) systems. Development of liquid hydrogen indirectly cooled $\mathrm{MgB}_{2}$ superconducting HVDC cables especially for computer data centres and whole electric ships present ideal candidates for early implementation [22]. Hydrogen's use as a coolant, as well as an energy carrier, will spin off new research and developments in superconducting materials and efficient energy use greatly increasing the scale of superconductivity applications.

Concerning hydrogen-cooled superconducting materials used to manufacture practical conductors, there are two main categories of which some irreversibility lines are represented in Fig. 3:

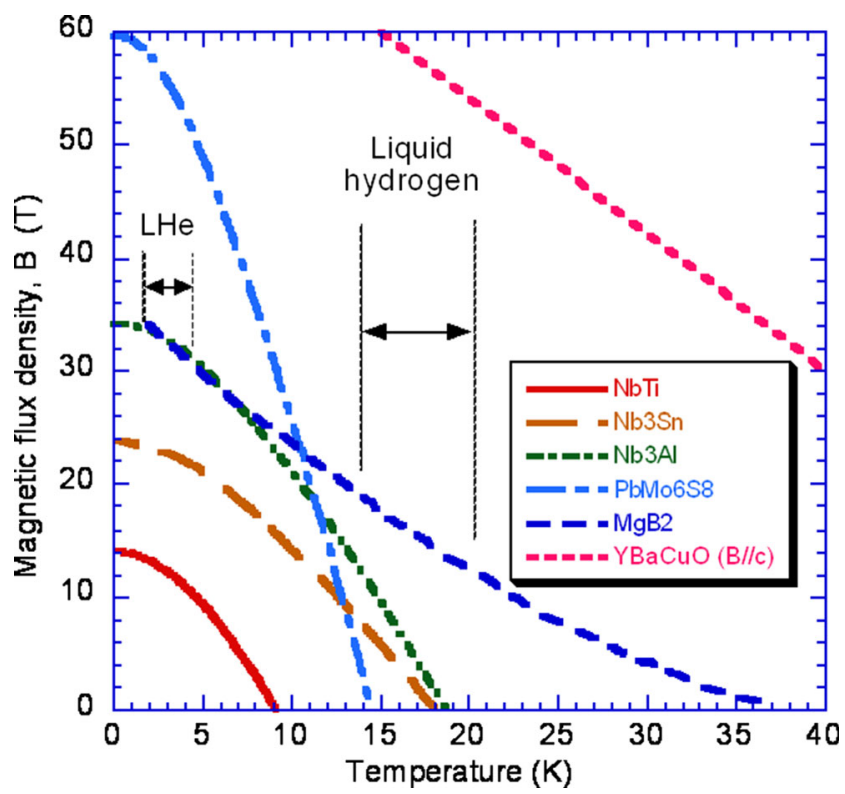

Fig. 3 Plot of magnetic induction irreversibility, $B_{\text {irr }}$, versus temperature, $T$, for low-critical-temperature superconductors, mediumcritical-temperature $\mathrm{MgB}_{2}\left(T_{\mathrm{c}}=39 \mathrm{~K}\right)$ and high-critical-temperature superconductor $\mathrm{YBa}_{2} \mathrm{Cu}_{3} \mathrm{O}_{7}$. (Magnetic flux density, $B$, is parallel to crystallographic c-direction of the superconducting $\mathrm{YBa}_{2} \mathrm{Cu}_{3} \mathrm{O}_{7}$ layer) 
- Medium-temperature superconductors: e.g. $\mathrm{MgB}_{2}$ $\left(\mathrm{T}_{\mathrm{c}}=39 \mathrm{~K}\right), \mathrm{SmO}_{1-\mathrm{x}} \mathrm{F}_{\mathrm{X}} \mathrm{FeAs}\left(T_{\mathrm{c}}=53 \mathrm{~K}\right)$

- High-temperature superconductors: $\mathrm{YBa}_{2} \mathrm{Cu}_{3} \mathrm{O}_{7}\left(T_{\mathrm{c}}=\right.$ $91 \mathrm{~K}),(\mathrm{Bi}, \mathrm{Pb})_{2} \mathrm{Sr}_{2} \mathrm{Ca}_{2} \mathrm{Cu}_{3} \mathrm{O}_{x},\left(T_{\mathrm{c}}=117 \mathrm{~K}\right)$ and $\mathrm{HgBa}_{2} \mathrm{Ca}_{2} \mathrm{Cu}_{3} \mathrm{O}_{\mathrm{x}}\left(T_{\mathrm{c}}=134 \mathrm{~K}\right)$

\section{Indirect Liquid Hydrogen Cooling}

Considering liquid hydrogen safety, direct cooling can only be handled by highly specialised organisations and companies, but indirect liquid hydrogen cooling, $\mathrm{iLH}_{2}$, can be a viable option for a decentralised economy. In $\mathrm{iLH}_{2}$ installations, a helium gas exchanger can be used, transferring cooling power of the hydrogen bath at $\sim 20 \mathrm{~K}$ to the desired cryomagnetic installation [23, 24].

A pertinent example of indirect cooling by liquid nitrogen is given by McDonald et al. [25] who designed a cooling system for a $15 \mathrm{~T}$ pulsed copper solenoid magnet to a desired temperature of $30 \mathrm{~K}$ in order to reduce the resistance of the $\mathrm{Cu}$ and thereby reducing the power requirements of the system. The design as proposed cooled the magnet via a closed helium loop circulated through a heat exchanger filled with liquid hydrogen from a storage Dewar. The piping and instrumentation diagram is shown in Fig. 4.

Helium was chosen as the circulating gas due to the unsuitability of hydrogen in operating circumstances where there was a chance of ignition through internal shorting of magnets. A cost comparison was then carried out to determine the viability of hydrogen for use as a liquid coolant. Economical calculations of cooling efficiency of the large 15-T electromagnetic non-superconducting device, supported by a study on testing for the scheduled purpose build installation in the USA conducted for $\mathrm{LHe}, \mathrm{LH}_{2}$ and $\mathrm{LN}_{2}$, show very clearly that cooling directly by helium or neon is 70 times and 100 times more expensive than indirect cooling by $\mathrm{LH}_{2}$ [25]. Taking into account the price of 11 of $\mathrm{LH}_{2} \sim £ 0.5$, LHe $\sim £ 12$ and $\mathrm{Ne} \sim £ 150$ and also the fact that hydrogen is the element that is both an energy carrier and cryogenic coolant, the choice of $\mathrm{LH}_{2}$ as a cryogenic cooling medium for cryomagnetic applications is rather apparent.

Development of efficient and cost-effective helium gas cryopumps is a paramount task for the implementation of $\mathrm{iLH}_{2}$ technology to the market and making the synergy between superconducting applications and liquid hydrogen a reality.

Currently, the hydrogen cryogenic gas circulator (e.g. Cryo-pump and CryoFan designs) is not very well developed in industry and as such only one product is readily available, the CryoZone CryoFan range (Fig. 5) [23]. This design introduces a direct thermal link through the impeller shaft at cryogenic temperatures to the motor assembly at ambient temperatures. This design necessitates a shaft seal, which must be able to withstand cryogenic temperatures and high pressures, as gas cooling requires a pressurised working gas in order to be effective.

Ongoing research aims to develop the concept of a magnetically coupled motor/impellor "CryoMagFan" which maintains a complete, uninterrupted vacuum envelope to reduce heat loss, prevent helium leakage and also allow for a higher operating pressure.

The face-on or axial-type magnetic coupling was chosen due to the ability to achieve higher coupling strength
Fig. 4 Piping and instrumentation schematic for 15-T Cu PSM indirect cooling. The design utilised two stages of cooling: the first preliminary stage of cooling provided by $\mathrm{LN}_{2}$ to $77 \mathrm{~K}$ and the second stage of cooling provided by $\mathrm{LH}_{2}$. The location of the compressed helium gas circulator is located above the bottom $\mathrm{LH}_{2}$ tank. This pump circulates GHe through the closed loop between the heat exchanger that is immersed in the $\mathrm{LH}_{2}$ dewar and magnet coil [25]

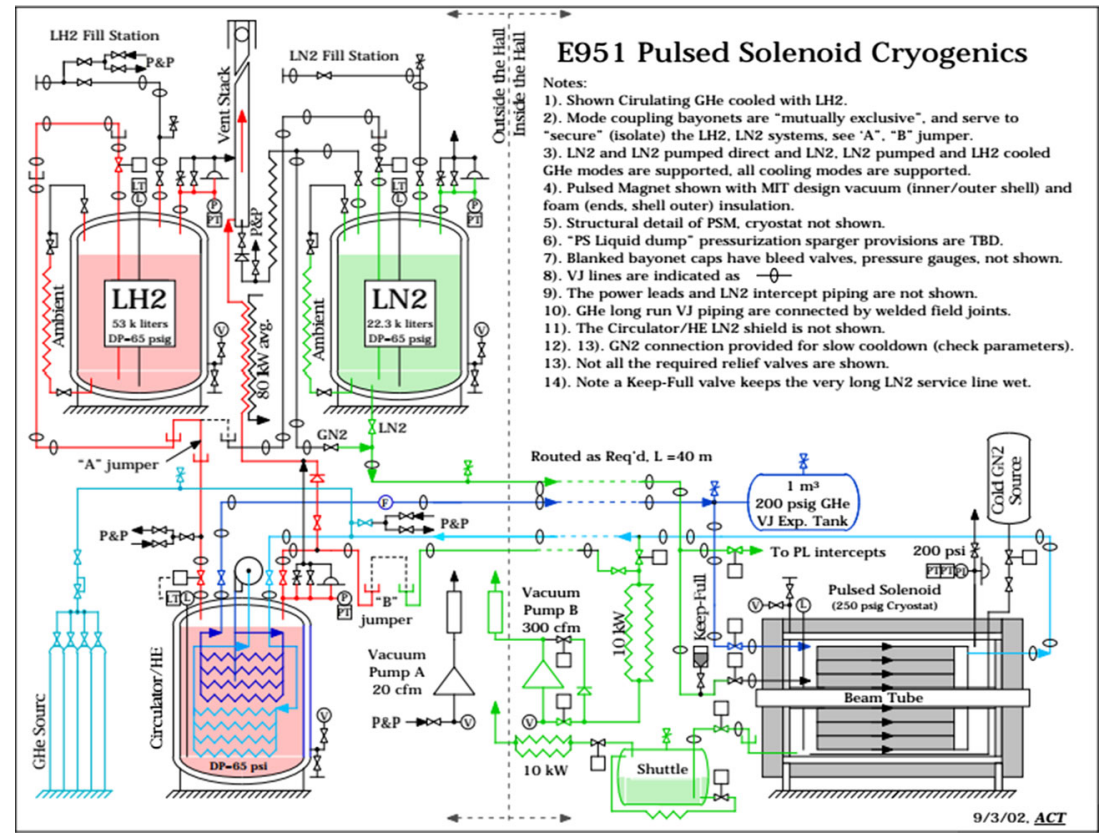


Fig. 5 a) A range of Cryozone cryofans [23]; b) (1) Schematic helium gas cooling system of Noordenwind CryoFan by Cryozone; (2) superconducting application such as

electromagnet, bearing, etc.; (3)

He heat exchanger; and (4) closed cycle cryocooler head or $\mathrm{LH}_{2}$ cryostat. (Pictures reproduced with permission of Cryozone) a)

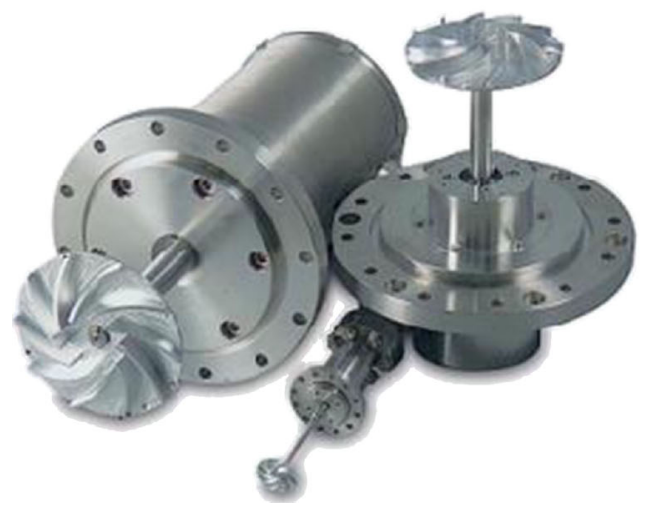

b)

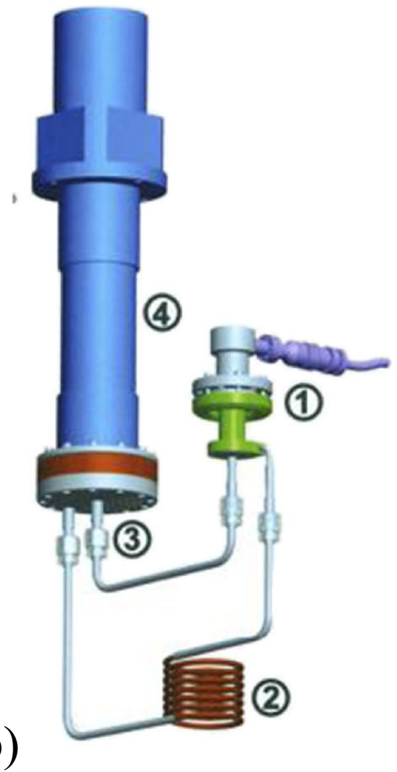

at greater separation distances-different types of couplings are discussed later in the report. This greater coupling force allows for a thicker insulation layer, which is a critical component in any cryogenic system. A prototype was manufactured as depicted in Fig. 6.

As an aside, there is an economic case to be made for cooling of fusion magnet systems via new temperature superconductor (HTS) materials in the 15-20 K range cooled indirectly by hydrogen using closed loop compressed helium gas, a similar way as proposed by McDonald et al. [25] for pulse copper magnets. Ongoing research in the replacement of niobium superconducting magnets with the high HTS $\mathrm{YBa}_{2} \mathrm{Cu}_{3} \mathrm{O}_{7}$ (yttrium barium copper oxide) could facilitate indirect cooling by $\mathrm{LN}_{2}$ through $\mathrm{GHe}$ closed loop. Therefore, indirect cooling by hydrogen $\mathrm{iLH}_{2}$ of the future fusion reactor system would reduce costs through elimination of He consumption and energy costs associated with achieving LHe temperatures [24].

\section{Decentralised Hydrogen Economy-Hydrogen Cryomagnetics}

\subsection{Building Infrastructure $\mathrm{iLH}_{2}$ Concept Implementation}

It is clear that with the expected rising prices of helium, an alternative cryogenic is required. Hydrogen is not suitable

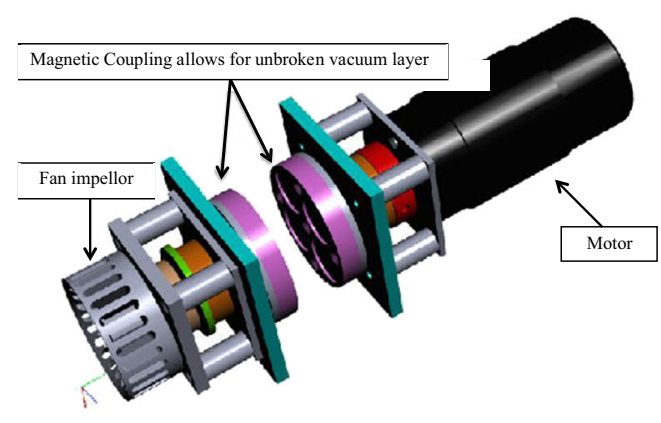

a)

Fig. 6 a) A novel concept of magnetically coupled CryoMagFan which enables unbroken vacuum insulation layer; b) Expanded view of CryomagFan prototype in situ. The insulation layer is observed encapsulating the fan/magnet element, which is submersed within the cooling gas 
as a direct coolant due to fire hazards, and neon is not suitable as a result of high costs. However, a suitable solution is the indirect cooling of a closed helium loop with hydrogen that would allow the cooling of the magnet without having to continuously buy diminishing helium resources, with expected rising prices.

Considering all the above-mentioned reasons for using the indirect cooling of technical superconductors with liquid hydrogen in conjunction with a compressed closed helium gas loop, we would like to discuss one hospital-related system that is generic for the decentralised hydrogen economy, where synergy between hydrogen generation, liquefaction, storage and use can have a very well justified place.

In Fig. 7, hydrogen is delivered to the hydrogen cell from renewable or zero- $\mathrm{CO}_{2}$-emitting sources where it is liquefied outside the buildings, and from the storage reservoir providing indirect cooling via a helium closed loop to both MRI magnets and to the superconducting bearings of a flywheel energy storage system, via DC cables for the data centre for an uninterruptable power supply by closed loop of helium gas circulation.

\subsection{Hospital Hydrogen Integration System}

Figure 8 shows a decentralised scenario for the use of hydrogen in meeting future energy storage, energy demand and cryogenic requirements in conjunction with a hospital environment. The choice of hydrogen production is by renewable and low-carbon emission methods as the world is seeking to lower carbon emissions in an attempt to mitigate climate change. The importance of the hydrogen industry in enabling other industries can be identified with the production of carbon for the carbon industry as a product of microwave plasma reforming (MPR) and the production of liquid hydrogen providing $20 \mathrm{~K}$ for cryogenic applications enabling the superconducting industry.

The use of liquefied natural gas (LNG) as the method of transport for natural gas is used as a result of the growing importance of LNG for transport due to reduced costs and the capability of transporting and storing larger volumes. The secondary use of LNG in the liquefaction of hydrogen is illustrated; however, this is only suitable when hydrogen is being liquefied on-site the LNG plant. The conventional use of natural gas for electricity generation supplying the grid is also included. The hydrogen both compressed and liquefied in this decentralised hydrogen scenario is produced from wind energy using the electrolysis of water and natural gas using microwave plasma reactors. The compressed hydrogen can be used in the transport sector and also for domestic heating and electricity supply. Only heating applications are suitable when solid oxide fuel cells (SOFC) are used due to their high operating temperatures. Heating applications are not considered in this scenario as these fuel cells are currently being researched and developed. The use of liquid hydrogen is the main focus as a result of hydrogen cryomagnetics being capable of enabling the superconducting industry as it is facing resource problems due to the price increase of helium. From Fig. 8, liquid hydrogen is also suitable for transport applications for use in cars, e.g. BMW and buses, and these vehicles utilise liquid hydrogen instead of compressed hydrogen. This decentralised production of hydrogen can be used for domestic, transport and industrial applications, making it a cryogenic that can be used both as an energy carrier and a cryogenic medium.

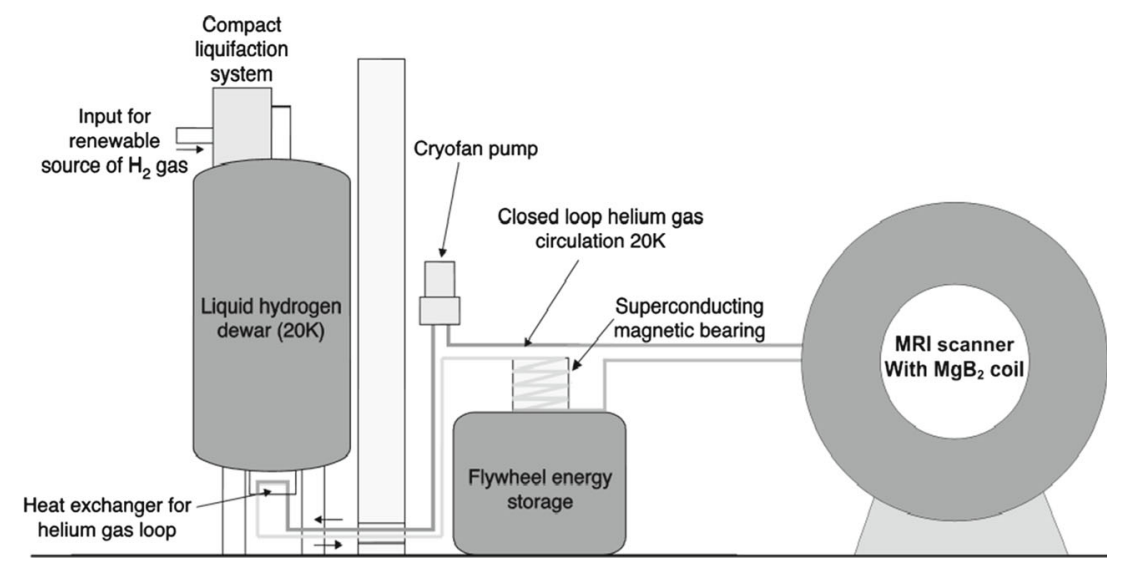

Fig. 7 Building infrastructure $\mathrm{iLH}_{2}$ concept of MRI and FES superconducting bearing cooled indirectly by $\mathrm{LH}_{2}$ using a closed-loop $\mathrm{He}$ circuit. $\mathrm{LH}_{2}$ storage dewar located external to the hospital. Indirect cooling of a fully superconducting $\mathrm{MgB}_{2} / \mathrm{YBaCuO}$ bearing [26] using a liquid hydrogen dewar and gHe CryoFan heat exchanger. Helium gas is pumped around a closed loop with one end cooled to $20 \mathrm{~K}$ via a heat exchanger connected to a liquid hydrogen dewar. This setup is under design and construction stage and is going to be a part of a liquid hydrogen laboratory in the new Department of Materials Science and Metallurgy building, University of Cambridge (building completed in 2013) 


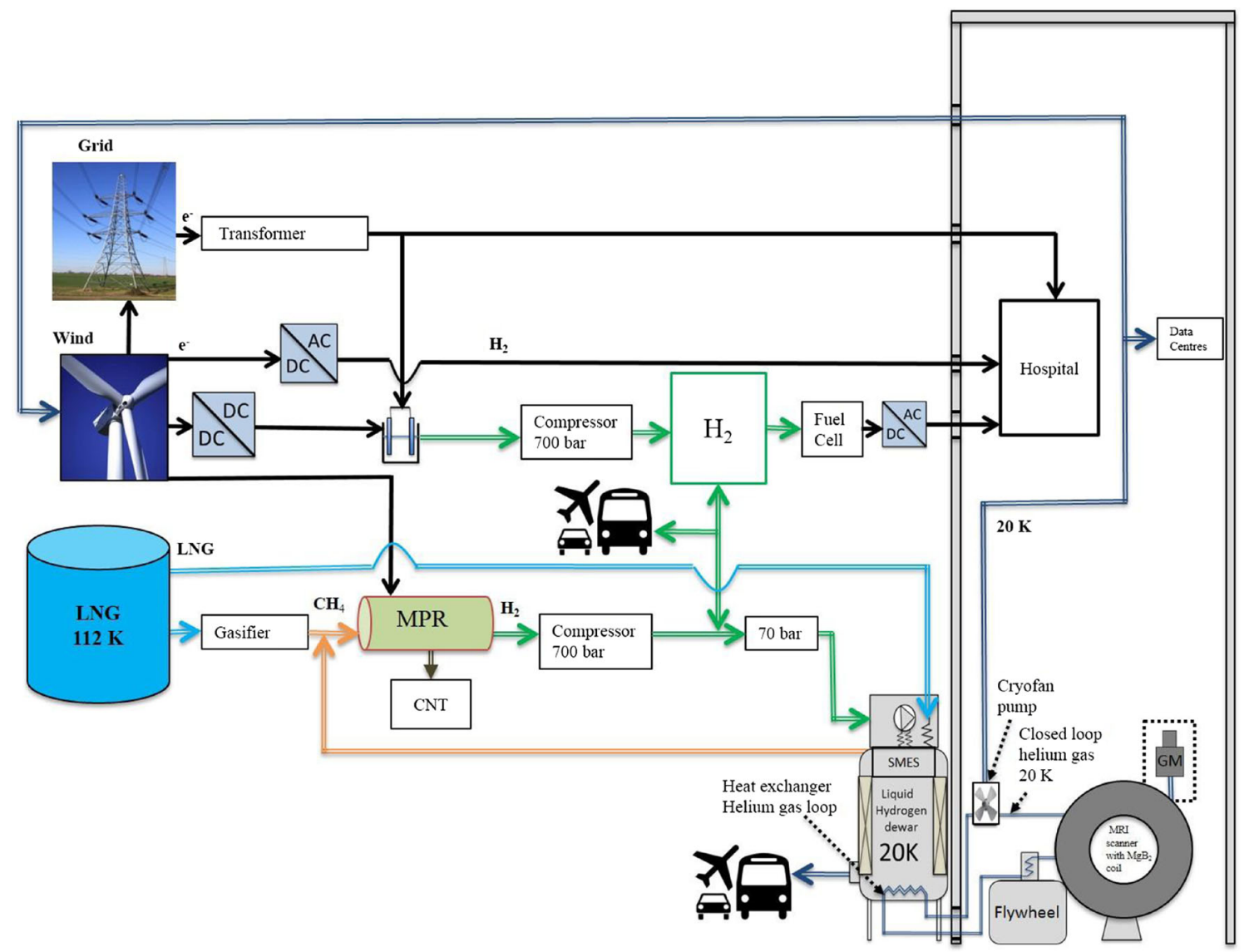

Fig. 8 Decentralised production of hydrogen via zero- $\mathrm{CO}_{2}$ emission processes and uses as an energy carrier, energy storage medium and cooling cryogenic medium. The actual superconducting devices to benefit from the synergy with $\mathrm{iLH}_{2}$ are SMES, Flywheel, MRI, DC cables for datacentres and homopolar wind generators. The hydrogen cryomagnetic superconductivity applications presented in the right corner of the figure are identical to the building infrastructure concept presented in Fig. 7
As the world is moving towards a low-carbon economy, the production of hydrogen from carbon-free technologies will be important. Microwave plasma reforming is an emission-free method of hydrogen production forming hydrogen and valuable carbon nanoforms [27]. This indicates that the hydrogen industry can facilitate the growth of the carbon industry. Two valuable products are produced from this process. With the growth of the global wind industry, wind is another potential emission-free source of hydrogen production. The wind industry is expected to reduce the world's consumption of fossil fuels; however, with the intermittency of wind energy, the use of energy storage is necessary. Hydrogen can be produced and compressed at times where there is excess demand and sold at times in which electricity costs are high to ensure that profit is made. The superconducting industry is facing problems with regard to supply of a suitable and a cost-effective cryogenic medium. The use of liquid hydrogen $\left(\mathrm{LH}_{2}\right)$ will enable the development of the superconducting industry.

\subsection{System Dynamics Simulation}

Following the successful study by Cai and colleagues on helium availability from natural resources using system dynamics [28], we suggest that a similar approach can be used to research the synergy between hydrogen and superconducting applications. The use of professional system dynamics (SD) simulation software 'Vensim DSS' should allow the dynamics of hydrogen production to be integrated with wind energy and MPR in a decentralised set-up to be modelled and simulated to gather results to determine the performance of the hydrogen production systems. The formulation of our SD model is based on the preparatory schematic depicted in Fig. 9 with wind energy being used to meet demand and also supply excess energy to the energy storage systems, which also supply energy to meet demand. Grid energy is used to meet any demand not met by the renewable technologies and storage systems. Electrolysis and MPR of methane 


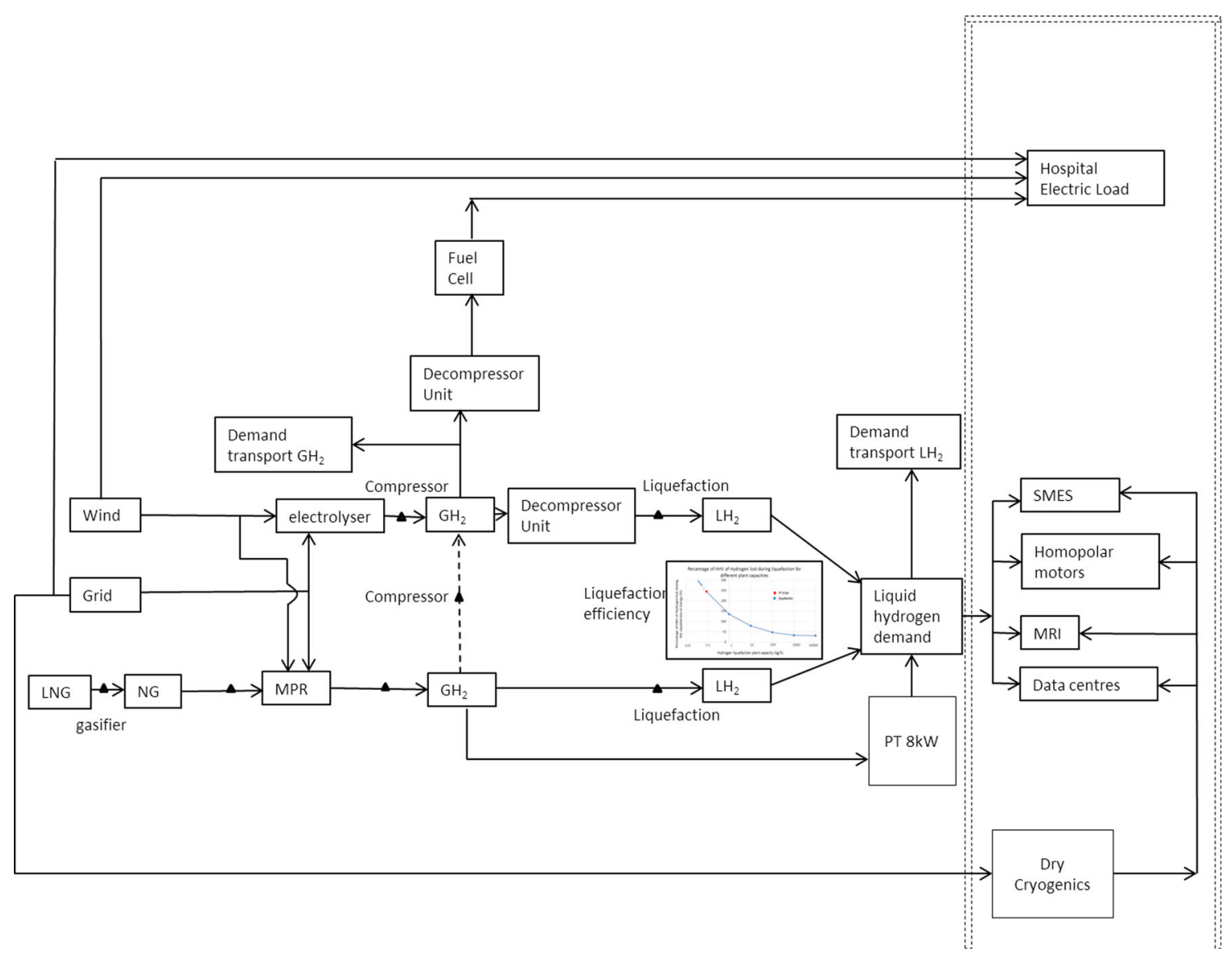

Fig. 9 Schematic showing the potential paths of wind energy, the potential electricity sources in meeting the electrical load and the potential methods for cryogenic applications. The graph shown in the

also are a potential liquid hydrogen source. System dynamics will allow different inputs of wind energy generated, demand and efficiencies of technologies and liquid hydrogen demand to be changed in the simulation and outputs of the system analysed. The approach allows real-world systems to be modelled and tested to see how they respond to the changing inputs. For example, the instantaneous output of the model to changing inputs can be tested in SyntheSim mode in the Vensim software.

The use of system dynamics should give insight into the amount of feedstock required for the microwave plasma reactor and the amount of energy required from the wind energy to produce liquid hydrogen. We expect it to be able to elucidate the effect that the amount of liquid hydrogen produced has on both costs and efficiencies shown in Fig. 10. Our initial modelling in Vensim has sought to identify the advantages (or otherwise) of the use of $\mathrm{LH}_{2}$ as a cryogenic medium for the superconducting industry. $\mathrm{LH}_{2}$ can be used for various applications. One important use in cryogenics is for MRI. The use of MRI in hospitals is vital, and there is a large cooling requirement as the power figure indicates the relationship used to model the cost and efficiency change of liquefaction that occurs, depending on the overall demand from transport and the superconducting industry

needed to magnetise the magnet is reduced as the temperature is reduced; however, little change is seen lower than a temperature of $30 \mathrm{~K}$ and so a cryogenic that has a boiling point lower than $30 \mathrm{~K}$ is suitable to cool the magnet. However, helium is the cryogenic of choice for MRI magnets and the superconducting industry, but due to escalating price and shortage of supply, indirect cooling by hydrogen is more suitable cryogenic medium solution. The superconducting industry in the model is divided into the MRI demand, use of liquid hydrogen for the indirect use of cooling DC cables and use in homopolar wind generators. The use of liquid hydrogen in transport is also considered with a fraction of the liquid hydrogen produced used for transport.

\subsection{Hydrogen Production, Use and Liquefaction Potential}

The model looks at two different aspects of hydrogen production and use. It looks at the use of hydrogen as a storage and energy carrier for electricity and also at the liquefaction efficiencies and the use of liquid hydrogen to meet demand. As the electrolysis of water is an emission-free method of 
Fig. 10 Percentage of the higher heating value of hydrogen lost during the liquefaction process for different plant capacities. The efficiency of pulse tube (PT) cryorefrigerator condenser-like liquefier is shown as a reference point

\section{Percentage of HHV of Hydrogen lost during liquefaction for different plant capacities}

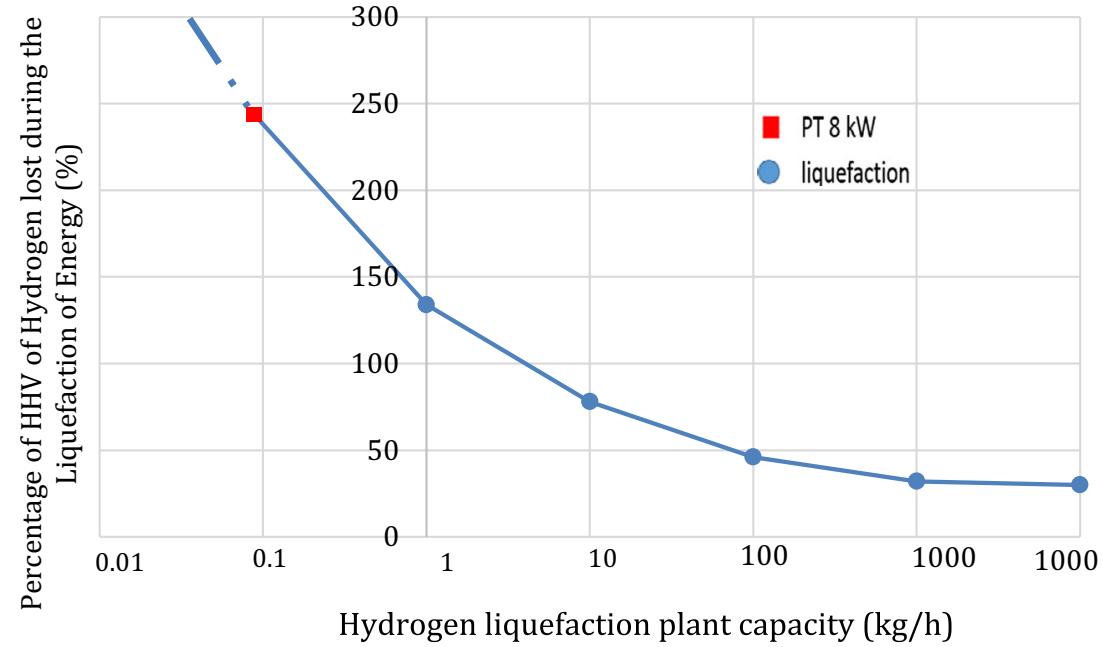

hydrogen production, it is considered in the model as a potential source for future storage and hydrogen needs. An advantage of the electrolysis of water in relation to hospitals arises from the production of oxygen as a product of electrolysis, which is required in hospitals. This gives decentralised hydrogen production via electrolysis for hospitals an added value. The problem that arises with using wind energy as a source of hydrogen results from it being an intermittent source of energy. If hydrogen is needed at a particular time and there are no adequate amounts available in storage, then hydrogen will need to be produced from another source. It is assumed that natural gas is available each day for the microwave plasma reactor to give a predictable production of hydrogen and liquefaction to meet demand. A large energy penalty is encountered (Fig. 10) when liquefying hydrogen, and the larger the quantity of hydrogen, the more efficient is the process. This means that for the efficient production of hydrogen, large amounts of

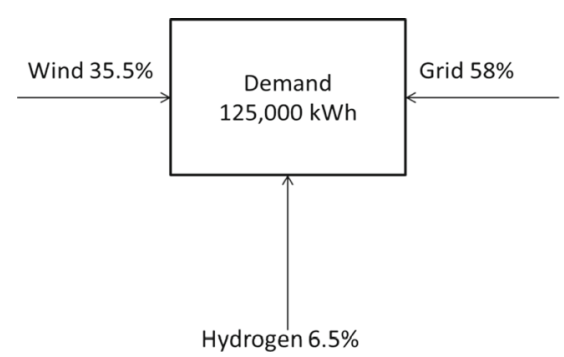

Fig. 11 Exemplary hospital sub-unit demand being met by wind energy, stored energy and grid energy. Hospital Demand for one building $20 \%$ of overall demand over a 72 hour period of the Addenbrookes Hospital in Cambridge hydrogen must be available for liquefaction. This potentially poses problems for the production of liquid hydrogen via intermittent wind energy. Using natural gas for hydrogen production results in the use of a fossil fuel, making this source of hydrogen unsustainable and not a long-term solution to global energy needs. Wind and other renewable energy are not suitable for large liquefaction plants as a result of unpredictable fluctuation in wind energy.

It is clear that even if hydrogen production and its applications are proven to be cost-effective, the success of the hydrogen economy both gaseous and liquid depends on the support from government policies.

When storing liquid hydrogen, losses can occur as a result of the low boiling temperature of liquid hydrogen $\left(T_{\text {boil }}=20 \mathrm{~K}\right)$, so the demand for liquid hydrogen must be present before a large liquefaction plant will be build.

For our SD modelling, we assume that liquid hydrogen is only produced if the demand is present, the demand is constant per hour throughout the day and the overall demand can be changed. As a result of the energy penalties that are present when liquefying hydrogen, extra hydrogen is produced to account for the overall energy loss as shown in Fig. 10. In the model, the demand is 6 $\mathrm{kg} / \mathrm{h}$ over the 24 hour period; however, to actually produce this amount, $18.2 \mathrm{~kg} / \mathrm{h}$ will have to be produced that has a corresponding energy efficiency of $33 \%$, allowing $6 \mathrm{~kg} / \mathrm{h}$ to be produced and the required energy available. SMES is a method of energy storage that can act as a source of backup electricity. In the model, it is only considered as a potential liquid hydrogen demand and does not supply electricity to the hospital.

Gaseous hydrogen use is also considered as a store for wind energy, as hydrogen is a promising future solution 
to an increase of wind energy into the electricity supply. Wind can therefore provide power directly to the electrical load, can supply electricity for the electrolysis of water to produce hydrogen and can also provide electricity to the microwave plasma reactor for operation. The use of wind energy to supply the microwave plasma reactor would lower any emissions resulting from the use of grid energy. For the hospital, if available wind energy can be used directly, any stored energy can be reconverted to electricity at times of low wind, and grid electricity can act as a backup if neither source can meet overall demand. The main aim of hydrogen storage is to store energy that would be otherwise wasted and in doing so, increase the amount of renewable electricity available and displace conventional fossil fuel power stations as a source of electricity. The use of hydrogen storage to displace grid energy is depicted in Fig. 11.

\section{Conclusions}

In the presented case, the use of indirect cooling is necessitated by safety concerns over storage of hydrogen within the building envelope. However, fundamental concerns over the cost and availability of helium which are already hampering progress for direct cooling dictate that superconductor cooling will be increasingly catered for via indirect closed loop systems in order to conserve helium and reduce operating costs.

The development of effective low-loss and highefficiency helium gas CryoMagFans is of paramount importance for safe use of cryogenic applications in everyday life.

The decentralised production of hydrogen via electrolysis for a hospital has the potential to supply the hospital with an energy store, electricity, liquid hydrogen for cryogenic applications and oxygen.

With regard to energy storage and supply, the demand from hospitals is large, and so a large decentralised system would have to be built, maybe in combination with a biomass CHP to reduce overall emissions and become independent from grid energy.

If a building with an approximate demand of 41,600 $\mathrm{kWh} /$ day was powered by the wind-hydrogen system, then the grid dependency of the building over a 72 hour period will be $58 \%$, with $35.5 \%$ from wind and $6.5 \%$ from hydrogen storage using actual wind data.

As hospitals are often composed of different buildings, it could be assumed that a decentralised system would aid one building in reducing grid dependency.

The larger the role of $\mathrm{LH}_{2}$ generation in the hospital environment, the less important is the cost of the cryogenic coolant for superconducting applications.
Electrolysis is not the only decentralised solution as the plasma microwave reformer can be twice more costeffective in hydrogen generation than wind energy hydrogen via electrolysis. Considering microwave plasma reforming as a zero- $\mathrm{CO}_{2}$ emission process with the possibility of producing valuable carbon nanoforms as a by-product, it appears to be a very attractive decentralised hydrogen generation solution for liquefaction.

We are currently advancing our understanding of these issues via the use of system dynamics modelling of systems associated with liquid hydrogen and hydrogen cryomagnetics.

Acknowledgment The authors wish to thank Dr M. Wozniak for his contribution to the design and manufacture of the CryoMagFan prototype.

Open Access This article is distributed under the terms of the Creative Commons Attribution License which permits any use, distribution, and reproduction in any medium, provided the original author(s) and the source are credited.

\section{References}

1. US Energy Information Administration, International Energy Outlook 2013, (2013). Available: http://www.eia.gov/forecasts/ieo/ pdf/0484 (2013).pdf. Accessed 3 Feb 2014

2. Wind power - emerging markets drive growth, Reinforced Plastics, Vol. 56(4), 2012, Pp. 32-33, ISSN 0034-3617, doi:10.1016/S0034-3617(12)70079-6

3. SBC Energy Institute. Electricity storage, (2013). Available: http://www.sbc.slb.com/SBCInstitute/Publications/ /media/ Files/SBC\%20Energy\%20Institute/SBC\%20Energy\%20Institute_ Electricity_Storage\%20Factbook_vf.ashx . Accessed 3 Feb 2014

4. Argonne National Lab. Available: http://www.dis.anl.gov/news/ HydrogenMarkets.html. Accessed 3 Feb 2014

5. MarketsandMarkets, press release relating to report Global Hydrogen Generation Market by Merchant \& Captive Type, Distributed \& Centralized Generation, Application and Technology - Trends \& Forecasts (2011-2016). Available: http://www. marketsandmarkets.com/PressReleases/hydrogen.asp. Accessed 12 Jan 2014

6. Saur, G.: Wind-to-hydrogen project: electrolyser capital cost study, (2008). Available: http://citeseerx.ist.psu.edu/viewdoc/ download?doi=10.1.1.173.5360\&rep=rep1\&type=pdf. Accessed 4 Feb 2014

7. Carmo, M., Fritz, D.L., Mergel, J., Stolten, D.: A comprehensive review on PEM water electrolysis. Int. J. Hydrog. Energy 38(12), 4901-4934 (2013). doi:10.1016/j.ijhydene.2013.01.151. ISSN 0360-3199

8. Steward, D., Saur, G., Penev, M., Ramsden, T.: lifecycle cost analysis of hydrogen versus other technologies for electrical energy storage, (2009). Available: http://www.nrel.gov/docs/ fy10osti/46719.pdf. Accessed 14 Feb 2014

9. Suzuki, J., Takahashi, K.: DOE hydrogen and fuel cells program record, (2009). Available: http://www.hydrogen.energy.gov/ pdfs/9013_energy_requirements_for_hydrogen_gas_compression.pdf. Accessed 4 Feb 2014 
10. US Department of Energy Efficiency and Renewable Energy. Comparison of fuel cell technologies. Available: http://www1.eere.energy.gov/hydrogenandfuelcells/fuelcells/pdfs/ fc_comparison_chart.pdf. Accessed 3 Feb 2014

11. Office of Energy Efficiency and Renewable Energy, Hydrogen Properties, (2001). Available: http://www1.eere.energy. gov/hydrogenandfuelcells/tech_validation/pdfs/fcm01r0.pdf. Accessed 16 Jan 2014

12. Wade, A.A.: Costs of storing and transporting hydrogen, (1998). Available: http://www.nrel.gov/docs/fy99osti/25106.pdf. Accessed 16 Jan 2014

13. Yasuhiro, M., Hiromi, H., Takakazu, S., Shinichi, N.: Design of SMES system with liquid hydrogen for emergency purpose. IEEE Trans. Appl. Supercond. 17(2) (2007)

14. Sander, M., Gehring, R., Neumann, H.: LIQHYSMES-A 48 GJ torodial MgB2 SMES for buffering minute and second fluctuations. IEEE Trans. Appl. Supercond. 3 (2013)

15. Glowacki, B.A., Nuttall, W.J., Clarke, R.H. IEEE Trans. Appl. Supercond. 23, 0500113 (2013)

16. Nuttall, W.J., Clarke, R., Glowacki, B.A.: The Future of Helium as a Natural Resource, Routledge. Taylor \& Francis Group Ltd, Oxford (2012). ISBN: 978-0-415-57697-0

17. Glowacki, B.A., Nuttall, W.J.: Hydrogen as a fuel and as a coolant-from the superconductivity perspective. Journal of Energy Science 1, 15-29 (2010)

18. Glowacki, B.A., Majoros, M., Vickers, M., Evetts, J.E., Shi, Y., McDougall, I.: Supercond. Sci. Technol. 14, 193 (2001)

19. Guina Energy PTY Ltd. Available: http://www.guinarnd.com/ index.php/history. Accessed 15 May 2014

20. Glowacki, B.A., Nuttall, W.J.: J. Phys. Conf. Ser. 97, 012333 (2008)

21. Makida, Y., Hirabayashi, H., Shintomi, T., Nomura, S.: IEEE Trans. Appl. Supercond. 17, 2006 (2007)
22. Cheadle, M.J., Woźniak, M., Bromberg, L., Glowacki, B.A., Jiang, X., Zeng, R., Minervini, J.V., Brisson, J.G.: IEEE Trans. Appl. Supercond. 23, 6200805 (2013)

23. Cryozone. Available: http://www.cryozone.nl/products/ cryogenic-fans/all/cryogenic-fans/. Accessed 15 May 2014

24. Clarke, R.H., Glowacki, B.A.: Indirect hydrogen versus helium or nitrogen cooling for fusion cryogenic and magnet systems. In: Proceedings of 2rd International Cryogenic Engineering Conference International Cryogenic Materials Conference 2010, pp. 347-352, Wroclaw (2010)

25. McDonald, K.T., Iarocci, M., Kirk, H.G., Mulholland, G.T., Titus, P.H., Weggel, R.J.: Use of He Gas Cooled by Liquid Hydrogen with a 15-T Pulsed Copper Solenoid Magnet, Technical / Economic Report August 15, (2002) Page 1-5 E951; also presented at ICEC 23 - ICMC 2010, 19-23 July 2010, Wrocław, Poland (to be published in Adv Cryo. Eng.)

26. Patel, A., Hopkins, S.C., Giunchi, G., Figini Albisetti, A., Shi, Y., Palka, R., Cardwell, D., Glowacki, B.A.: The use of an $\mathrm{MgB}_{2}$ hollow cylinder and pulse magnetized (RE)BCO bulk for magnetic levitation applications. IEEE Trans. Appl. Supercond. 23(3), 6800604 (2013)

27. Juda, K., Clayton, A., Glowacki, B.A.: The influence of microwave plasma power on the degree of natural gas conversion to carbon nanoforms article in book. In: Proceedings of the 2nd Global Congress on Microwave Energy Applications (eds. R. L. Schulz and D.C. Fox), pp. 307-319, 48 hr Books, ISBN: 0978622219 (2013). 2nd Global Congress on Microwave Energy Applications (2GCMEA 2012), Long Beach, California (2012)

28. Cai, Z., Clarke, R.H., Glowacki, B.A., Nuttall, W.J., Ward, N.: Ongoing ascent to the helium production plateau insights from system dynamics resources policy 35, 77-89 (2010) 